OPEN ACCESS

Edited by:

Zhongji Meng,

Hubei University of Medicine, China

Reviewed by:

Qian Zhou,

The First Affiliated Hospital of Sun Yat-Sen University, China

Baoju Wang,

Huazhong University of Science and

Technology, China

${ }^{*}$ Correspondence:

Yu Chen

chenyuzy@zju.edu.cn

${ }^{\dagger}$ These authors have contributed equally to this work

Specialty section: This article was submitted to

Viral Immunology,

a section of the journal

Frontiers in Immunology

Received: 16 May 2021 Accepted: 08 October 2021 Published: 27 October 2021

Citation:

Lou B, Ma G, LVF, Yuan $Q$, Xu F, Dong $Y$, Lin S, Tan $Y$, Zhang $J$ and Chen Y (2021) Baseline Quantitative Hepatitis B Core Antibody Titer Is a

Predictor for Hepatitis $B$ Virus Infection Recurrence After Orthotopic Liver Transplantation.

Front. Immunol. 12:710528. doi: 10.3389/fimmu.2021.710528

\section{Baseline Quantitative Hepatitis B Core Antibody Titer Is a Predictor for Hepatitis B Virus Infection Recurrence After Orthotopic Liver Transplantation}

\author{
Bin Lou ${ }^{1,2,3 \dagger}$, Guanghua $\mathrm{Ma}^{1 \dagger}$, Feifei $\mathrm{LV}^{1}$, Quan Yuan ${ }^{4}$, Fanjie $\mathrm{Xu}{ }^{1}$, Yuejiao Dong ${ }^{1}$, \\ Sha Lin ${ }^{1}$, Yajun Tan ${ }^{1}$, Jie Zhang ${ }^{1}$ and Yu Chen ${ }^{1,2,3^{*}}$
}

\footnotetext{
${ }^{1}$ Department of Laboratory Medicine, The First Affiliated Hospital, College of Medicine, Zhejiang University, Hangzhou, China, ${ }^{2}$ Key Laboratory of Clinical In Vitro Diagnostic Techniques of Zhejiang Province, Hangzhou, China, ${ }^{3}$ Institute of Laboratory Medicine, Zhejiang University, Hangzhou, China, ${ }^{4}$ National Institute of Diagnostics and Vaccine Development in Infectious Disease, School of Life Science, Xiamen University, Xiamen, China
}

Objective: Hepatitis B virus (HBV) reinfection is a serious complication that arise in patients who undergo hepatitis B virus related liver transplantation. We aimed to use biomarkers to evaluate the HBV reinfection in patients after orthotopic liver transplantation.

Methods: Seventy-nine patients who underwent liver transplantation between 2009 and 2015 were enrolled, and levels of biomarkers were analyzed at different time points. Cox regression and receiver operating characteristic (ROC) curves of different markers at baseline were used to analyze sustained hepatitis B surface antigen (HBsAg) loss. The Kaplan-Meier method was used to compare the levels of the biomarkers.

Results: Among the 79 patients, 42 sustained HBsAg loss with a median time of 65.2 months (12.0-114.5, IQR 19.5) after liver transplantation and 37 patients exhibited HBsAg recurrence with a median time of $8.8(0.47-59.53$, IQR 19.47) months. In the ROC curve analysis, at baseline, $4.25 \log _{10} \mathrm{IU} / \mathrm{mL}$ qHBcAb and $2.82 \log _{10} \mathrm{IU} / \mathrm{mL}$ qHBsAg showed the maximum Youden's index values with area under the curves (AUCs) of 0.685and 0.651, respectively. The Kaplan-Meier method indicated that $\mathrm{qHBsAg}$ and quantitative antibody against hepatitis B core antigen ( $\mathrm{qHBcAb}$ ) levels in the two groups were significantly different $(p=0.031$ and 0.006 , respectively). Furthermore, the Cox regression model confirmed the predictive ability of $\mathrm{qHBCAb}$ at baseline ( $\mathrm{AUC}=0.685$ ).

Conclusion: Lower pretransplantation $\mathrm{qHBCAb}$ is associated with HBV infection. The baseline concentration of qHBcAb is a promising predictor for the recurrence of HBV in patients undergoing liver transplantation and can be used to guide antiviral treatment for HBV infection.

Keywords: qHBcAb, liver transplantation, HBV recurrence, sustained HBV loss, qHBsAg 


\section{INTRODUCTION}

Hepatitis B virus (HBV) infection is a global health problem. Chronic hepatitis $\mathrm{B}(\mathrm{CHB})$ is a major disease that threatens human health, especially in southeast Asia and Africa. According to clinical statistics, the global prevalence of hepatitis B surface antigen (HBsAg) was $3.9 \%$ in 2016, corresponding to 291 million infections (1). Additionally, nearly $25 \%$ of chronic HBV carriers develop terminal stage liver-related diseases including chronic hepatitis, cirrhosis, and primary hepatocellular carcinoma, causing 0.8 million deaths annually $(2,3)$. Liver transplantation (LT) is an established treatment option for serious liver disease caused by HBV infection. The data from the European Liver Transplant Registry database (ELTR) verified that viral hepatitis B was the most common indication (9.8\%) of LT from 2007 to 2017 (4). HBV recurrence is a risk factor for patients who have undergone LT and leads to grievous failure of treatment and poor prognosis $(5,6)$. The HBV reinfection rate was higher than $90 \%$ (7) and the 2-year survival rate was only $50 \%$ (8) before the application of immune globulin and antiviral drugs. In recent years, the therapeutic strategy of nucleos(t)ide analogs (NAs) combined with immune globulin has proven useful in preventing HBV reinfection after $\operatorname{LT}(9,10)$. However, this strategy does not completely protect against future recurrence of $\mathrm{HBV}$ infection. HBV reinfection is not only dependent on residual viral infection in extrahepatic organs, but also individual immune responses. Therefore, it is necessary to find satisfactory biomarkers that reflect specific immune responses to $\mathrm{HBV}$ to predict the recurrence of HBsAg.

Antibody against hepatitis $\mathrm{B}$ core antigen $(\mathrm{HBcAb})$ is a widespread biomarker in patients with $\mathrm{HBV}$ infection and can be present in current or previous infections. Routine serum screening for $\mathrm{HBcAb}$ and other $\mathrm{HBV}$-related biomarkers has been performed in some countries with a high HBV infection rate. Nevertheless, for some patients, $\mathrm{HBcAb}$ may be the only serological marker of $\mathrm{HBV}$ infection in serum $(11,12)$, and "HBcAb only" status may reflect occult HBV infection (13). Additionally, $\mathrm{HBcAb}$ alone can be used to evaluate the risk for $\mathrm{HBV}$ reactivation in patients who have received antiviral treatment that may lead to immunosuppression, patients with human immunodeficiency virus (HIV) infection or chronic hepatitis $\mathrm{C}$ virus (HCV) infection $(14,15)$.

One study revealed that $\mathrm{HBcAb}$ plays a valuable role in $\mathrm{CHB}$ disease by inhibiting or clearing HBV (16). Thus, patients with a high titer of $\mathrm{HBcAb}$ before therapy had a stronger acquired immune response that was related to a satisfactory outcome after antiviral treatment (17).

Owing to the inferior performance of the competitive immunoassay, current commercially available $\mathrm{HBcAb}$ assays have a narrow linearity range. A novel immunoassay for qualitative $\mathrm{HBcAb}$ based on the double-antigen sandwich enzyme-linked immunosorbent assay method has demonstrated sensitivity and specificity (18).

In this retrospective longitudinal study, we aimed to analyze the clinical value of $\mathrm{qHBcAb}, \mathrm{qHBsAg}$ and other viral marker concentrations, focusing on the outcome of patients who underwent LT.

\section{MATERIALS AND METHODS}

\section{Patients}

Overall, 363 patients who underwent HBV-related LT between 2009 and 2015 at the First Affiliated Hospital, College of Medicine, Zhejiang University were enrolled in this study. The patients selected were also negative for HCV infection, HIV infection, and other chronic diseases. Serum samples were collected and stored at $-80^{\circ} \mathrm{C}$ until use. This study was reviewed and approved by the Ethics Committees of the First Affiliated Hospital, College of Medicine, Zhejiang University. This study followed the 1964 Helsinki Declaration and its later amendments. Prior informed consent was obtained from the enrolled patients or their legal guardians.

\section{Post-Transplant Therapy}

Antiviral prophylaxis was administered to all recipients, and they were treated with NAs in combination with low-dose hepatitis B immunoglobulin (HBIG). Briefly, 2000 IU HBIG was intravenously injected during the anhepatic phase, followed by 800 IU daily intramuscular administration for the first week and then weekly for 3 weeks, and monthly thereafter.

All patients received a Tacrolimus based steroid-sparing or steroid-free immunosuppression regimen. Tacrolimus and mycophenolate mofetil (750 mg every $12 \mathrm{~h}$ ) were administered from the first post-operative day. The target blood trough level of tacrolimus was $7-10 \mathrm{ng} / \mathrm{mL}$ for the first postoperative month and was aimed at 5-7 $\mathrm{ng} / \mathrm{mL}$ thereafter.

\section{Measurement of qHBcAb Concentration in Serum}

The $\mathrm{qHBcAb}$ level in serum was measured using a newly developed double-sandwich immunoassay (100-100 $000 \mathrm{IU} / \mathrm{mL}$; Wantai, China), calibrated using the World Health Organization standard (NIBSC, UK) $(19,20)$. QHBcAb was measured at baseline, with the sought time of HBsAg-positive status before LT being at least two weeks, and then at different time points after LT.

\section{Measurement of qHBsAg, qHBeAg, HBcAb and HBV DNA Concentrations in the Serum}

Serum levels of qHBsAg, qHBeAg and $\mathrm{HBcAb}$ were measured using a chemiluminescence microparticle immunoassay (CMIA) on an Abbott Architect I4000 automated analyzer (Abbott Laboratories, Chicago, IL, USA). HBV DNA levels in serum were measured using Qiagen PCR kits (Hilden, Germany) on ABI 7500 qRT-PCR System (ABI Laboratories, USA) according to the manufacturer's instructions. The linear detection range was $3-7 \log _{10} \mathrm{IU} / \mathrm{mL}$, with a correlation coefficient of the standard curve $>0.995$. Biomarkers were measured before LT and at different time points after LT.

\section{Statistical Analysis}

Chi-squared and Mann-Whitney $U$ tests were performed as appropriate. The accuracy of serum $\mathrm{qHBcAb}$ and $\mathrm{qHBsAg}$ levels 
predicting HBsAg recurrence was determined by applying the receiver operating characteristic (ROC) curve analysis. HBV reinfection was assessed using Kaplan-Meier survival analysis. The risk of HBV reinfection after LT was determined using the Cox regression model. Statistical significance was set at $p<0.05$. Statistical analysis and presentation were performed using SPSS software (version 23.0; SPSS, Chicago, IL, USA). Graphical analysis and data presentation were performed using GraphPad Prism version 5.0 and $\mathrm{R}$ version 4.0 (R Foundation).

\section{RESULTS}

\section{Characteristics of Patients}

A total of 363 patients were enrolled in this study, 79 patients were selected retrospectively, and 284 patients were excluded including 44 patients with HBsAg-negative status, 53 patients with underlying diseases or other infectious diseases, 18 patients receiving an HBV-positive graft, 17 patients who died post LT, 5 patients who underwent retransplantation and 147 patients without sufficient serum samples or integrated follow-up time points (Supplementary Figure 1). Among the 79 enrolled patients, 37 exhibited HBsAg recurrence with a median time of 8.8 (0.47 - 59.53, IQR 19.47) months, and 42 sustained HBsAg loss with a median follow-up time of 65.2 months (12.0 - 114.5, IQR 19.5) after LT.

The clinical characteristics of patients at baseline are shown in Table 1 for patients who retained sustained HBsAg loss or achieved HBsAg recurrence during the follow-up period. There were no significant differences in sex, age and treatment received between the two groups $(p=0.672,0.089$ and 0.515 , respectively). The characteristics of $\mathrm{qHBcAb}$ and $\mathrm{qHBsAg}$ were significantly different between the groups $(p=0.037$ and 0.023 , respectively). Compared with $\mathrm{HBcAb}$ measured by Abbott Architect CMIA (indirect method), the difference in $\mathrm{qHBcAb}$ measured by double-sandwich immunoassay was more significant between the observed groups ( $p=0.023$ vs 0.743 ). The titers of HBV DNA, qHBeAg, alanine aminotransferase (ALT) and aspartate aminotransferase (AST) were not significantly different among the compared groups $(p=0.394$, $0.732,0.453$ and 0.146 , respectively).

\section{Kinetics of qHBcAb and qHBsAg in HBsAg Recurrence and Sustained HBsAg Loss Groups}

As shown in Figure 1, during baseline to 1 week after LT, both qHBsAg and $\mathrm{qHBcAb}$ were significantly decreased in both sustained HBsAg loss group and HBsAg recurrence group $(p<$ 0.001 and $p<0.05)$. However, the changes in $\mathrm{qHBcAb}$ and qHBsAg in the two groups were not significantly different after 1 week. At each time point between baseline and 24 weeks after LT, the titer of $\mathrm{qHBcAb}$ in the sustained HBsAg loss group was higher than that in the HBsAg recurrence group.

\section{Comparison of Potential Risk Factors at Baseline Using the Cox Regression Model}

To further evaluate the potential risk factors associated with HBV recurrence after LT, Cox regression analysis was performed for age, sex, ALT, HBV DNA, qHBsAg, qHBeAg and qHBcAb. As shown in Table 2 and Supplementary Figure 2, the qHBcAb level at baseline was a strong predictor for HBsAg recurrence after LT, with both the univariate and multivariate analyses (crude HR: $0.40 ; 95 \% \mathrm{CI}$ : $0.20-0.78 ; p=0.01$ vs adjusted HR 0.42; 95\% CI: 0.19-0.94; $p=0.04$ ).

TABLE 1 | Characteristics of enrolled patients at baseline.

\begin{tabular}{|c|c|c|c|}
\hline Characteristics & $\begin{array}{l}\text { HBsAg recurrence } \\
\text { in follow-up time } \\
\qquad(n=37)\end{array}$ & $\begin{array}{c}\text { Sustained HBsAg loss } \\
\text { after LT } \\
(n=42)\end{array}$ & $p$ \\
\hline Male, n (\%) & $33(89 \%)$ & $37(88 \%)$ & $0.672^{a}$ \\
\hline $\begin{array}{l}\text { Age (years) at baseline before } \\
\text { liver transformation, median (range) }\end{array}$ & $54(28-67)$ & $48(29-66)$ & $0.089^{b}$ \\
\hline Treatment received & & & $0.515^{a}$ \\
\hline LAM/HBIG & 17 (45.95\%) & 22 (52.38\%) & \\
\hline ADV/LAM/HBIG & $10(27.03 \%)$ & $8(19.05 \%)$ & \\
\hline ETV/HBIG & $6(16.22 \%)$ & $4(9.52 \%)$ & \\
\hline ETV/LAM/HBIG & $4(10.81 \%)$ & $8(19.05 \%)$ & \\
\hline HBV related disease before $L T$ & & & $0.293^{a}$ \\
\hline $\mathrm{HCC}$ & 22 (59.46\%) & $20(47.62 \%)$ & \\
\hline Non-HCC & 15 (40.54\%) & 22 (52.38\%) & \\
\hline qHBsAg $\left(\log _{10} \mathrm{IU} / \mathrm{mL}\right)$, median (range) & $2.80(-0.31-3.95)$ & $2.34(-0.89-4.22)$ & $0.037^{C *}$ \\
\hline $\mathrm{qHBeAg}\left(\log _{10} 0.18 \mathrm{PEIU} / \mathrm{mL}\right)$, median (range) & $-0.36(0-1.57)$ & $-0.39(0-1.46)$ & $0.732^{c}$ \\
\hline $\mathrm{HBcAb}(\mathrm{S} / \mathrm{CO})$ & $10.38(0.22-47.27)$ & $10.73(1.88-14.16)$ & $0.743^{C}$ \\
\hline qHBcAb (log $10 \mathrm{IU} / \mathrm{mL})$, median (range) & $3.51(1.06-4.85)$ & $4.01(2.12-5.12)$ & $0.023^{c *}$ \\
\hline HBV DNA (positive\%) & 12 (32.43\%) & $10(23.81 \%)$ & $0.394^{a}$ \\
\hline ALT (IU/mL), median (range) & $39(14-1149)$ & $43(17-221)$ & $0.453^{C}$ \\
\hline AST (IU/mL), median (range) & $59(22-1535)$ & $53(21-355)$ & $0.146^{c}$ \\
\hline
\end{tabular}

LAM, lamivudine; ADV, adefovir; ETV, entecavir; HBIG, hepatitis B immunoglobulin; ALT, alanine aminotransferase; AST, aspartate aminotransferase; HCC, hepatocellular carcinoma. ${ }^{a}$ Chi-Square test. ${ }^{b}$ Independent samples $t$ test. ${ }^{c}$ Mann-Whitney $\cup$ test. ${ }^{*} p<0.05$. 
A

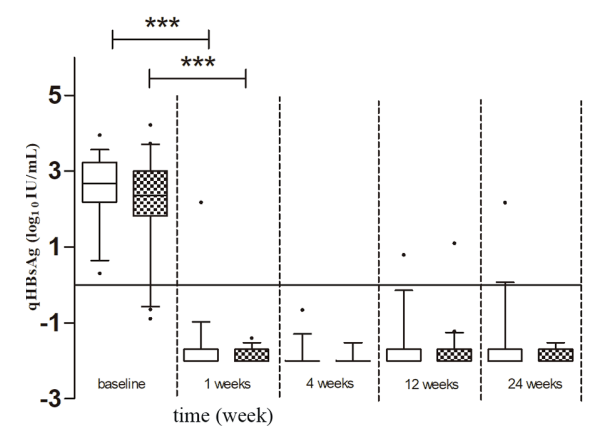

B

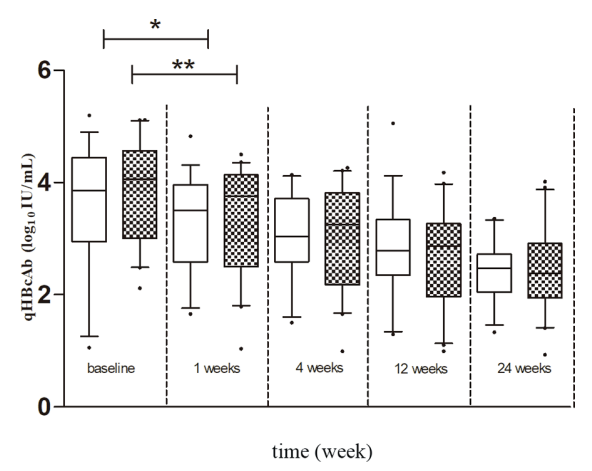

FIGURE 1 | (A) The Kinetics of qHBsAg in HBsAg recurrence and sustained HBsAg loss groups. (B) The Kinetics of qHBcAb in HBsAg recurrence and sustained HBsAg loss groups. Box plots showing the median, interquartile range and absolute range of qHBcAb and qHBsAg at baseline and at 1 week, 4 weeks, 12 weeks and 24 weeks in groups (blank box plots: HBsAg recurrence group; latticed box: sustained HBsAg loss group. ${ }^{*} p<0.05 ;{ }^{* \star} p<0.01 ;{ }^{* \star *} p<0.001$ ).

TABLE 2 | Risk factors for HBV recurrence after liver transplantation.

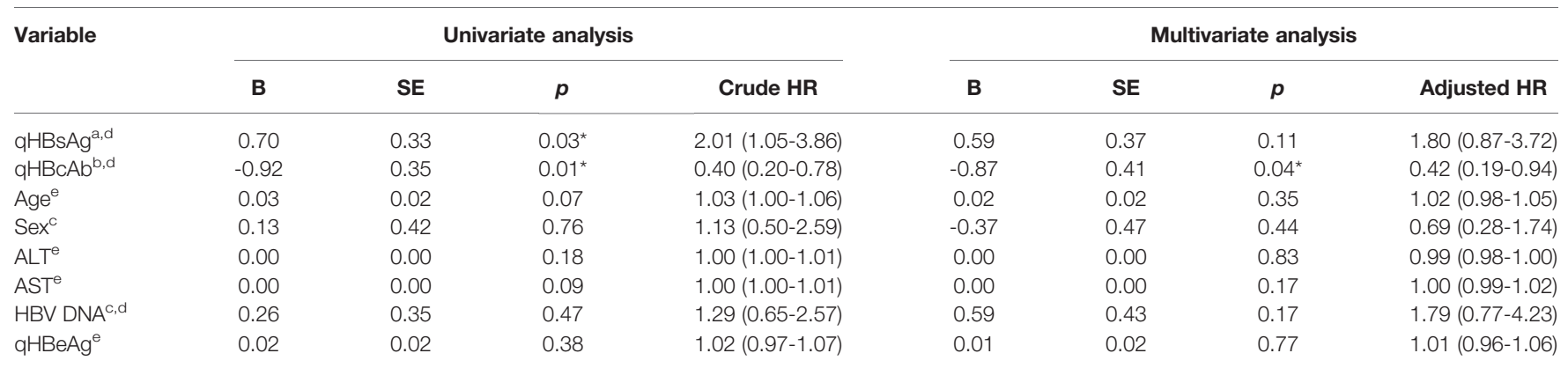

The variables included in the Cox regression analysis were age, sex (female vs male), and baseline ALT, HBV DNA, qHBsAg, qHBeAg and qHBcAb levels.

${ }^{a}$ qHBsAg $\leq 2.82$ vs $>2.82 \log 10 \mathrm{IU} / \mathrm{mL},{ }^{b} \mathrm{HBCAb}<4.25 \mathrm{vs} \geq 4.25 \log 10 \mathrm{IU} / \mathrm{mL},{ }^{\mathrm{C}} \mathrm{HBV} \mathrm{DNA} \leq 3.0 \mathrm{vs}>3.0 \mathrm{log} 10 \mathrm{IU} / \mathrm{mL},{ }^{d} \mathrm{Categ}$ orical variable, ${ }^{e} \mathrm{Continuous} \mathrm{variable,}{ }^{*} \mathrm{P}<0.05$.

\section{QHBcAb and qHBsAg as Predictors of HBsAg Recurrence}

The ability of qHBcAb and qHBsAg at baseline to predict HBsAg recurrence was analyzed using ROC curves to compare HBsAg recurrence and sustained HBsAg loss groups (Figures 2A, C). The area under the curves (AUCs) of $\mathrm{qHBcAb}$ and $\mathrm{qHBsAg}$ were 0.685 (95\% confidence interval, $0.577-0.799)$ and $0.651(95 \%$ confidence interval, 0.538 - 0.763), respectively. Using the Youden index (Figures 2B, D), we predicted that when qHBcAb was higher than $4.25 \log _{10} \mathrm{IU} / \mathrm{mL}$ and $\mathrm{qHBs} A g$ was lower than $2.82 \log _{10} \mathrm{IU} / \mathrm{mL}$, sustained HBsAg loss would occur more readily after LT.

\section{Combined qHBcAb With qHBsAg as Predictors of HBsAg Recurrence}

As shown in Figure 3, the combination of qHBsAg and $\mathrm{qHBcAb}$ was a stronger predictor of HBsAg recurrence than individual biomarkers at baseline. The cutoff values of $1.81 \log 10 \mathrm{IU} / \mathrm{mL}$ and $3.68 \log 10 \mathrm{IU} / \mathrm{mL}$ at baseline for $\mathrm{qHBsAg}$ and $\mathrm{qHBcAb}$, respectively, had sensitivity of $72.0 \%$, specificity of $62.2 \%$, PPV of $72.0 \%$ and NPV 62.2\%, with an AUC value of 0.727 (0.621, 0.833; 95\% CI) (Supplementary Table 1).

\section{Comparison of qHBcAb and qHBsAg at Baseline Using Kaplan-Meier Method}

Figures 4A, B show the comparison of HBsAg recurrence rates at the time of the last follow-up by Kaplan-Meier method. Patients who experienced high a risk of HBsAg recurrence had lower $\mathrm{qHBcAb}\left(\mathrm{qHBcAb}<4.25 \log _{10} \mathrm{IU} / \mathrm{mL}\right)$ and higher qHBsAg (qHBsAg > $2.82 \log _{10} \mathrm{IU} / \mathrm{mL}$ ) levels than those who achieved sustained HBsAg loss ( $p=0.006$ and 0.031 , respectively) (Figure 4).

\section{DISCUSSION}

$\mathrm{HBV}$ infection is associated with progression to hepatocellular carcinoma or other end-stage liver diseases, and LT is the only curative therapeutic method (21). However, increased liver transplantation failure rate and decreased patient survival rate in these patients are correlated with $\mathrm{HBV}$ reinfection and recurrence of hepatocellular carcinoma (22). HBIG and antiviral treatments are administered alone or in combination to prevent reinfection with HBV after LT (23). HBIG and nucleos(t)ide analogs can prevent reinfection with $\mathrm{HBV}$ 
A

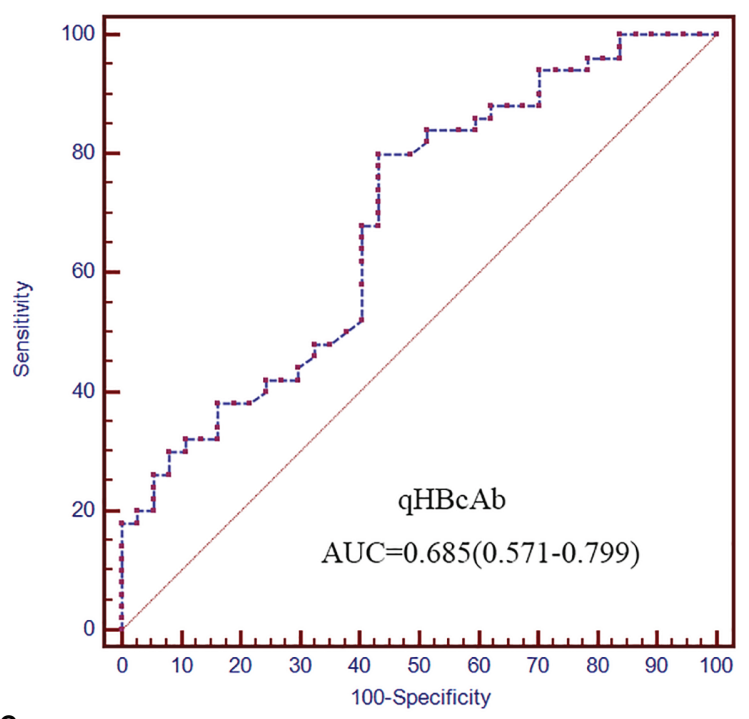

C

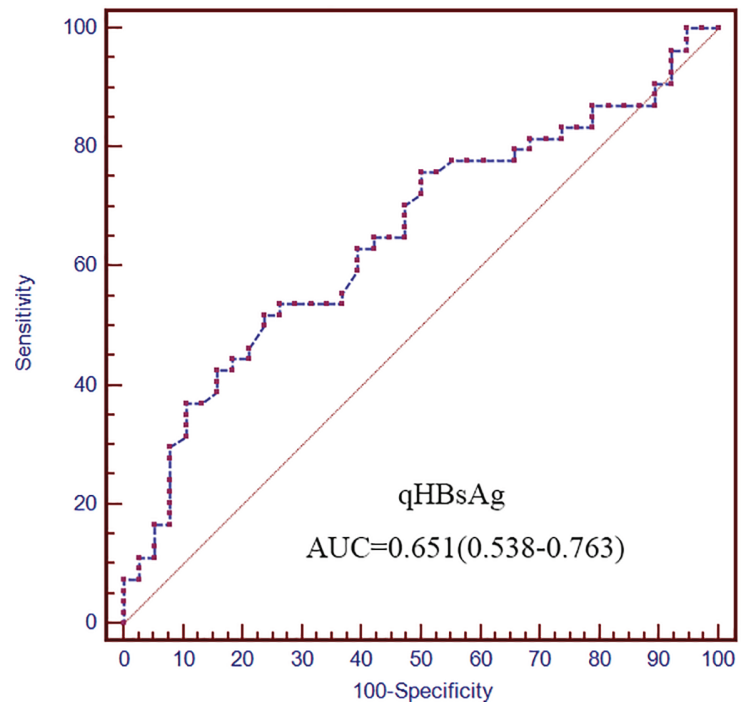

B

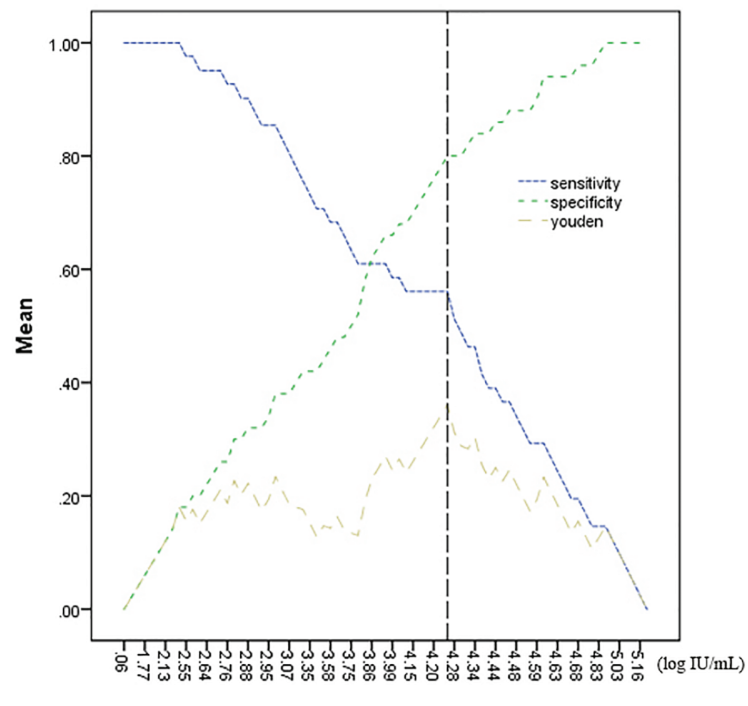

D

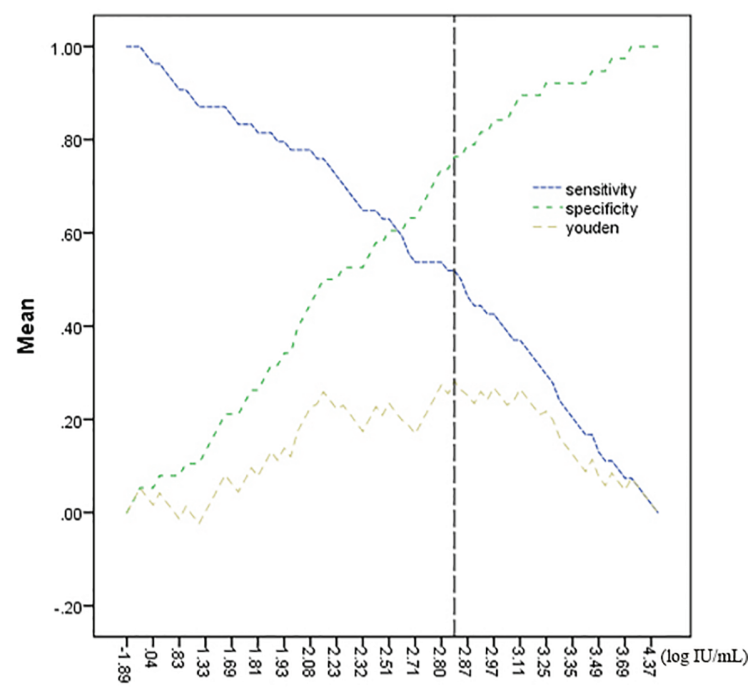

FIGURE 2 | QHBcAb and qHBsAg as predictors of HBsAg recurrence. (A, C). Receiver operating characteristic (ROC) analyses of $q \mathrm{HBcAb}$ and $\mathrm{qHBs} A \mathrm{~g}$ as predictors of HBsAg recurrence at baseline. (B, D). Curves of the specificity, sensitivity and Youden index for qHBcAb and qHBsAg. The position of the black vertical dotted line shows the maximum Youden index that was used to predict HBsAg recurrence.

through different mechanisms. HBIG eliminates circulating virus particles and induces antiviral and immune-mediated functions (24), while nucleos(t)ide analogs directly inhibit the process of viral reverse transcription, thus reducing viral load (25). The treatment of such patients aims to increase the "sustained HBsAg loss" rate during the period of follow-up. The HBV reinfection rate in patients post-LT was approximately $10 \%$ after they received combined prophylactic treatment with HBIG and NAs (26).

A newly developed assay based on double-sandwich immunoassay technology to measure $\mathrm{qHBcAb}$ showed higher sensitivity and specificity than those based on competitive/ inhibitory principles $(19,27)$. Several studies have focused on the use of $\mathrm{qHBcAb}$ to evaluate the antiviral therapeutic effect in patients with $\mathrm{CHB}$ disease. Yuan et al. revealed that $\mathrm{qHBcAb}$ was closely correlated with hepatic inflammatory activities and can serve as a new marker of antiviral treatment response in $\mathrm{CHB}$ patients $(17,28)$. However, the measurement of $\mathrm{qHBcAb}$ to determine the risk of HBsAg recurrence after LT has not been reported. This study aimed to analyze $\mathrm{qHBcAb}$ and other serological viral markers to identify the predictors of $\mathrm{HBsAg}$ recurrence after LT.

Here, ROC curve was used to predict HBsAg recurrence, we found that $\mathrm{qHBcAb}$ was a stronger predictor than $\mathrm{qHBsAg}$ of HBsAg recurrence after LT due to its larger AUC value. In addition, Cox regression analysis was performed to identify the 
A

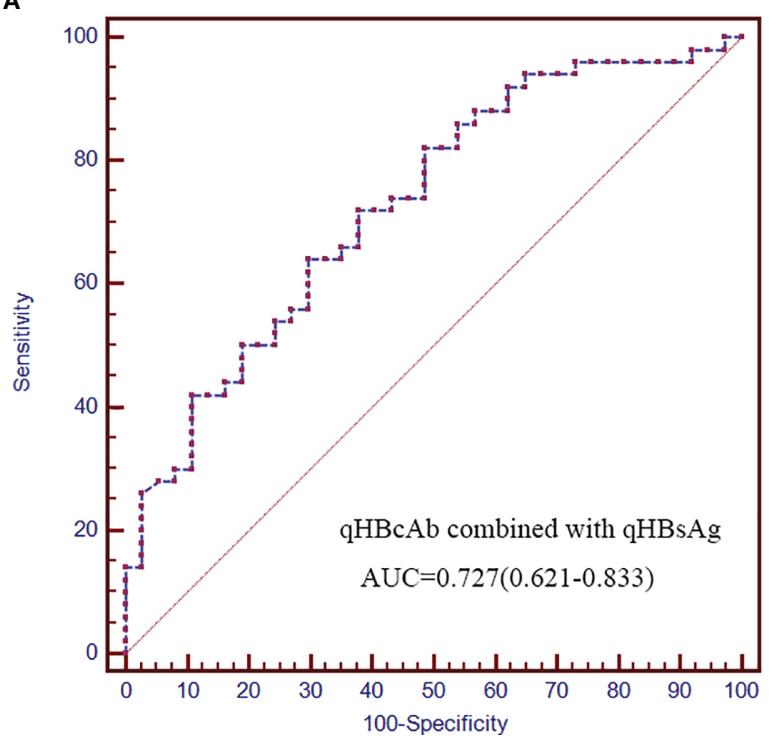

B

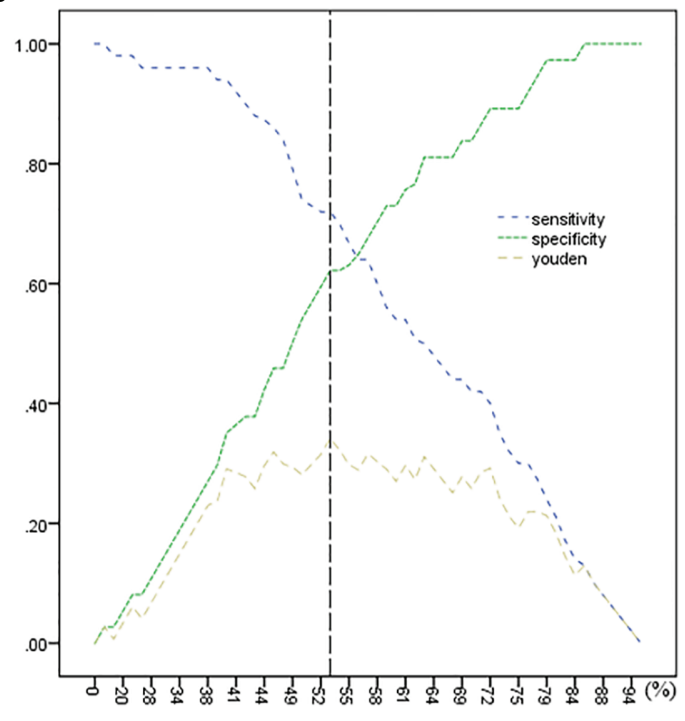

FIGURE 3 | Combined qHBcAb with qHBsAg as predictors of HBsAg recurrence. (A) The ROC curve of combined qHBcAb and qHBsAg at baseline. (B) Curves of the specificity, sensitivity and Youden index. The position of the black vertical dotted line shows the maximum Youden index that was used to predict HBsAg recurrence.

A

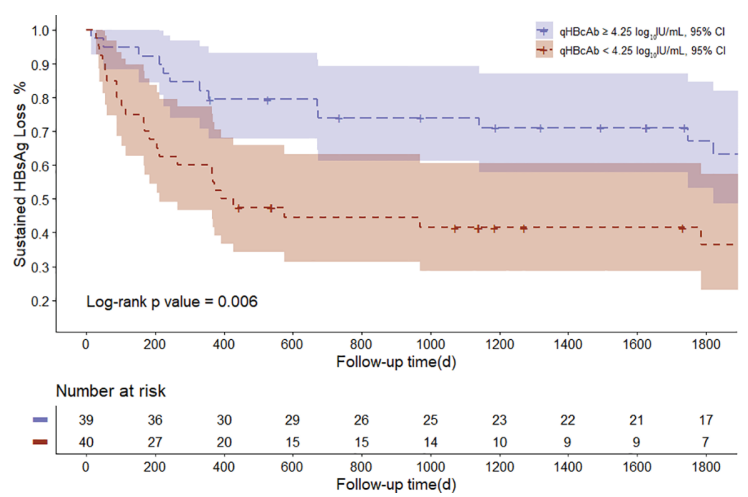

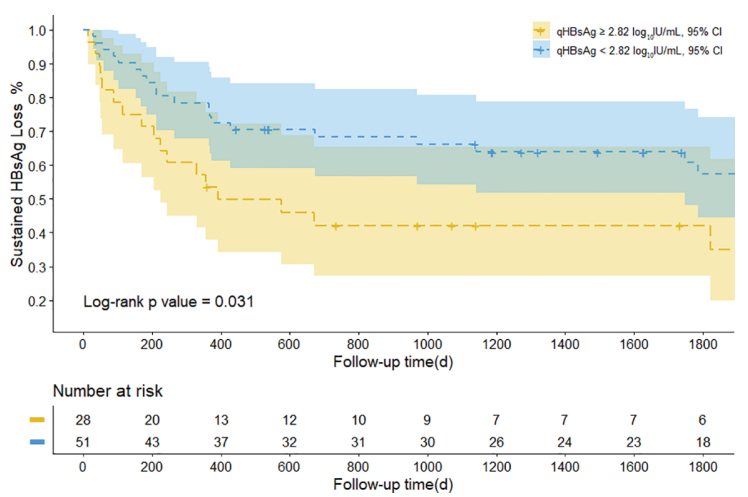

FIGURE 4 | The Kaplan-Meier curve of HBsAg conversion rates at the last time of follow-up. (A) The proportion of patients with sustained HBsAg loss according to qHBcAb at baseline. (B) The proportion of patients with sustained HBsAg loss according to qHBsAg at baseline.

potential risk factors for HBsAg recurrence after LT; qHBcAb and qHBsAg levels at baseline had more satisfactory HR scores than other markers, confirming the predictive ability of qHBcAb and $\mathrm{qHBsAg}$ for HBsAg recurrence after LT.

Furthermore, on the basis of cutoff values obtained from the $\mathrm{ROC}$ analysis at baseline, we used the different ranges of $\mathrm{qHBcAb}$ and qHBsAg levels to evaluate the sustained HBsAg loss rate by Kaplan-Meier analysis. We found that low $\mathrm{qHBcAb}$ and high qHBsAg levels were associated with HBV reinfection.

Circulating HBsAg correlates with the presence of covalently closed circular DNA (cccDNA) (29), and is a key marker of infection, a high HBsAg level indicates a high hepatitis B viral load in vivo. Therefore, patients with high serum qHBsAg titers are associated with high a probability of HBsAg recurrence after LT. However, the mechanism underlying the predictive value of $\mathrm{HBcAb}$ titer remains unclear. Chan et al. revealed that hepatitis $B$ core antigen ( $\mathrm{HBcAg})$, a viral nucleocapsid protein, is the most immunogenic component of HBV (30). Zgair et al. demonstrated that $\mathrm{HBCAb}$ plays an important role in inhibiting $\mathrm{HBV}$ through the hepatocytotoxic effect of anti$\mathrm{HBc}$-secreting $\mathrm{B}$ cells (16). Thus, a high $\mathrm{qHBcAb}$ level can represent a strong immune response to $\mathrm{HBV}$ and may be the reason for sustained HBsAg loss in patients with a high titer of $\mathrm{qHBcAb}$ at baseline. 
In this study, some novel results were obtained in LT patients from baseline to the last follow-up period, and a higher $\mathrm{qHBcAb}$ level before LT corresponded with sustained HBsAg loss. These conclusions may be meaningful for adjusting the duration of antiviral medicine usage after LT in patients with different $\mathrm{qHBcAb}$ and $\mathrm{qHBsAg}$ levels. However, this study has some limitations. First, the results were based on a small sample size, possibly limiting the accuracy of results. Second, related data on HBV genotype and mutation were not acquired; this may play potential roles during treatment (31-33). Third, the markers mentioned in this study were only serum markers, while other immunological markers and liver tissues were not analyzed. In summary, this is the first study to describe $\mathrm{qHBcAb}$ as a marker to predict the treatment response of LT patients. The measurement of $\mathrm{qHBcAb}$ could potentially assist surgeons in identifying patients who might benefit from specific antiviral treatments after LT.

\section{DATA AVAILABILITY STATEMENT}

The original contributions presented in the study are included in the article/Supplementary Material. Further inquiries can be directed to the corresponding author.

\section{ETHICS STATEMENT}

The studies involving human participants were reviewed and approved by Ethics Committees of the First Affiliated Hospital,

\section{REFERENCES}

1. Polaris Observatory Collaborators. Global Prevalence, Treatment, and Prevention of Hepatitis B Virus Infection in 2016: A Modelling Study. Lancet Gastroenterol Hepatol (2018) 3(6):383-403. doi: 10.1016/S2468-1253 (18)30056-6

2. Urabe A, Imamura $M$, Tsuge $M$, Kan H, Fujino H, Fukuhara T, et al. The Relationship Between HBcrAg and HBV Reinfection in HBV Related PostLiver Transplantation Patients. J Gastroenterol (2016) 52:366-75. doi: 10.1007/s00535-016-1240-y

3. McNaughton AL, D’Arienzo V, Ansari MA, Lumley SF, Littlejohn M, Revill P, et al. Insights From Deep Sequencing of the HBV Genome-Unique, Tiny, and Misunderstood. Gastroenterology (2019) 156(2):384-99. doi: 10.1053/ j.gastro.2018.07.058

4. Belli LS, Perricone G, Adam R, Cortesi PA, Strazzabosco M, Facchetti R, et al. Impact of DAAs on Liver Transplantation: Major Effects on the Evolution of Indications and Results. An ELITA Study Based on the ELTR Registry. J Hepatol (2018) 69(4):810-7. doi: 10.1016/j.jhep.2018.06.010

5. Xi ZF, Xia Q. Recent Advances in Prevention of Hepatitis B Recurrence After Liver Transplantation. World J Gastroenterol (2015) 21(3):829-35. doi: 10.3748/wjg.v21.i3.829

6. Chauhan R, Lingala S, Gadiparthi C, Lahiri N, Mohanty SR, Wu J, et al. Reactivation of Hepatitis B After Liver Transplantation: Current Knowledge, Molecular Mechanisms and Implications in Management. World J Hepatol (2018) 10(3):352-70. doi: 10.4254/wjh.v10.i3.352

7. Karliova M, Malago M, Trippler M, Valentin-Gamazo C, Rothaar T, Broelsch CE, et al. Seroconversion in Patients With Acute Hepatitis B Reinfection After Liver Transplantation With a Combined Treatment of Lamivudine and Hepatitis B Immune Globulin. Transplant Proc (2002) 34(8):3319-22. doi: 10.1016/S0041-1345(02)03558-3
College of Medicine, Zhejiang University and 1964 Helsinki declaration and its later amendments. The patients/participants provided their written informed consent to participate in this study.

\section{AUTHOR CONTRIBUTIONS}

YC and QY planned and designed the study. BL and GM performed the experiments and wrote the paper. FL and FX performed serum qHBsAg, qHBeAg, HBcAb, and HBV DNA measurement experiments. YD, YT, and JZ performed the serum $\mathrm{qHBcAb}$ measurement. All authors contributed to the article and approved the submitted version.

\section{FUNDING}

This work was supported by the National 863 Program for the Biological and medical technology (no. 2011AA02A100) and the National Key Programs for Infectious Diseases of China (no. 2012ZX10002005).

\section{SUPPLEMENTARY MATERIAL}

The Supplementary Material for this article can be found online at: https://www.frontiersin.org/articles/10.3389/fimmu.2021. 710528/full\#supplementary-material

8. Starzl TE, Demetris AJ, Van Thiel D. Liver Transplantation. $N$ Engl J Med (1989) 321(16):1092-9. doi: 10.1056/NEJM198910193211606

9. Angus PW, Patterson SJ, Strasser SI, Valentin-Gamazo C, Rothaar T, Broelsch CE. A Randomized Study of Adefovir Dipivoxil in Place of HBIG in Combination With Lamivudine as Post-Liver Transplantation Hepatitis B Prophylaxis. Hepatology (2008) 48(5):1460-6. doi: 10.1002/hep.22524

10. Steinmuller T, Seehofer D, Rayes N, Müller AR, Settmacher U, Jonas S, et al. Increasing Applicability of Liver Transplantation for Patients With Hepatitis B-Related Liver Disease. Hepatology (2002) 35(6):1528-35. doi: 10.1053/ jhep.2002.33681

11. Grob P, Jilg W, Bornhak H, Gerken G, Gerlich W, Günther S, et al. Serological Pattern "Anti-HBc Alone": Report on a Workshop. J Med Virol (2000) 62 (4):450-5. doi: 10.1002/1096-9071(200012)62:4<450::AID-JMV9>3.0.CO;2-Y

12. Weber B, Melchior W, Gehrke R, Doerr HW, Berger A, Rabenau H. Hepatitis B Virus Markers in Anti-HBc Only Positive Individuals. J Med Virol (2001) 64 (3):312-9. doi: 10.1002/jmv.1052

13. Vitale F, Tramuto F, Orlando A, Vizzini G, Meli V, Cerame G, et al. Can the Serological Status of Anti-HBc Alone Be Considered a Sentinel Marker for Detection of Occult HBV Infection? J Med Virol (2008) 80:577-82. doi: 10.1002/jmv.21121

14. Pérez-Rodríguez MT, Sopeña B, Crespo M, Rivera A, González del Blanco T, Ocampo A, et al. Clinical Significance of "Anti-HBc Alone" in Human Immunodeficiency Virus-Positive Patients. World J Gastroenterol (2009) 15:1237-41. doi: 10.3748/wjg.15.1237

15. Wedemeyer H, Cornberg M, Tegtmeyer B, Frank H, Tillmann HL, Manns $\mathrm{MP}$, et al. Isolated Anti-HBV Core Phenotype in Anti-HCV-Positive Patients is Associated With Hepatitis C Virus Replication. Clin Microbiol Infect (2004) 10(1):70-2. doi: 10.1111/j.1469-0691.2004.00771.x

16. Zgair AK, Ghafil JA, Al-Sayidi RH. Direct Role of Antibody-Secreting B Cells in the Severity of Chronic Hepatitis B. J Med Virol (2015) 87(3):407-16. doi: 10.1002/jmv.24067 
17. Fan R, Sun J, Yuan Q, Xie Q, Bai X, Ning Q, et al. Baseline Quantitative Hepatitis B Core Antibody Titre Alone Strongly Predicts HBeAg Seroconversion Across Chronic Hepatitis B Patients Treated With Peginterferon or Nucleos(T)Ide Analogues. Gut (2016) 65:313-20. doi: 10.1136/gutjnl-2014-308546

18. Li A, Yuan Q, Huang Z, Fan J, Guo R, Lou B, et al. Novel Double-Antigen Sandwich Immunoassay for Human Hepatitis B Core Antibody. Clin Vaccine Immunol (2010) 17(3):464-9. doi: 10.1128/CVI.00457-09

19. World Health Organization. WHO International Standard: First International Standard for Anti-Hepatitis B Core Antigen. Available at: http://www.nibsc.ac. uk/documents/ifu/95-522.pdf.

20. Song LW, Liu PG, Liu CJ, Zhang TY, Cheng XD, Wu HL, et al. Quantitative Hepatitis B Core Antibody Levels in the Natural History of Hepatitis B Virus Infection. Clin Microbiol Infect (2015) 21(2):197-203. doi: 10.1016/ j.cmi.2014.10.002

21. Terrault NA, Bzowej NH, Chang KM, Hwang JP, Jonas MM, Murad MH, et al. AASLD Guidelines for Treatment of Chronic Hepatitis B. Hepatology (2016) 63:261-83. doi: 10.1002/hep.28156

22. Saab S, Yeganeh M, Nguyen K, Durazo F, Han S, Yersiz H, et al. Recurrence of Hepatocellular Carcinoma and Hepatitis B Reinfection in Hepatitis B Surface Antigen-Positive Patients After Liver Transplantation. Liver Transplant (2009) 15(11):1525-34. doi: 10.1002/lt.21882

23. Katz LH, Tur-Kaspa R, Guy DG, Paul M. Lamivudine or Adefovir Dipivoxil Alone or Combined With Immunoglobulin for Preventing Hepatitis B Recurrence After Liver Transplantation. Cochrane Database Syst Rev (2010) 7):CD006005. doi: 10.1002/14651858.CD006005.pub2

24. Buti M, Mas A, Prieto M, Casafont F, González A, Miras M, et al. Adherence to Lamivudine After an Early Withdrawal of Hepatitis B Immune Globulin Plays an Important Role in the Long-Term Prevention of Hepatitis B Virus Recurrence. Transplantation (2007) 84(5):650-4. doi: 10.1097/01.tp. 0000277289.23677.0a

25. Schreibman IR, SchiffER. Prevention and Treatment of Recurrent Hepatitis B After Liver Transplantation: The Current Role of Nucleoside and Nucleotide Analogues. Ann Clin Microbiol Antimicrob (2006) 5:8. doi: 10.1186/1476-0711-5-8

26. Faria LC, Gigou M, Roque-Afonso AM, Sebagh M, Roche B, Fallot G, et al. Hepatocellular Carcinoma Is Associated With an Increased Risk of Hepatitis B Virus Recurrence After Liver Transplantation. Gastroenterology (2008) 134 (7):1890-9. doi: 10.1053/j.gastro.2008.02.064

27. Lou B, Fan J, Yuan Q, Yajun T, Li T, Jie Z, et al. Performance Evaluation of Three Different Immunoassays for Detection of Antibodies to Hepatitis B Core. Clin Chem Lab Med (2013) 51(2):e23-5.

28. Yuan Q, Song LW, Liu CJ, Li Z, Liu PG, Huang CH, et al. Quantitative Hepatitis B Core Antibody Level may Help Predict Treatment Response in
Chronic Hepatitis B Patients. Gut (2013) 62:182-4. doi: 10.1136/gutjnl-2012302656

29. Tout I, Loureiro D, Mansouri A, Soumelis V, Boyer N, Asselah T. Hepatitis B Surface Antigen Seroclearance: Immune Mechanisms, Clinical Impact, Importance for Drug Development. J Hepatol (2020) 73(2):409-22. doi: 10.1016/j.jhep.2020.04.013

30. Chan TT, Chan WK, Wong GL, Chan AW, Nik Mustapha NR, Chan SL, et al. Positive Hepatitis B Core Antibody Is Associated With Cirrhosis and Hepatocellular Carcinoma in Nonalcoholic Fatty Liver Disease. Am J Gastroenterol (2020) 115(6):867-75. doi: 10.14309/ajg.0000000000000588

31. Brunetto MR, Oliveri F, Colombatto P, Moriconi F, Ciccorossi P, Coco B, et al. Hepatitis B Surface Antigen Serum Levels Help to Distinguish Active From Inactive Hepatitis B Virus Genotype D Carriers. Gastroenterology (2010) 139 (2):483-90. doi: 10.1053/j.gastro.2010.04.052

32. Martinot-Peignoux M, Lapalus M, Laouénan C, Lada O, Netto-Cardoso AC, Boyer N, et al. Prediction of Disease Reactivation in Asymptomatic Hepatitis B E Antigen-Negative Chronic Hepatitis B Patients Using Baseline Serum Measurements of HBsAg and HBV-DNA. J Clin Virol (2013) 58(2):401-7. doi: 10.1016/j.jcv.2013.08.010

33. Betz-Stablein BD, Töpfer A, Littlejohn M, Yuen L, Colledge D, Sozzi V, et al. Single-Molecule Sequencing Reveals Complex Genome Variation of Hepatitis B Virus During 15 Years of Chronic Infection Following Liver Transplantation. J Virol (2016) 90(16):7171-83. doi: 10.1128/JVI. 00243-16

Conflict of Interest: The authors declare that the research was conducted in the absence of any commercial or financial relationships that could be construed as a potential conflict of interest.

Publisher's Note: All claims expressed in this article are solely those of the authors and do not necessarily represent those of their affiliated organizations, or those of the publisher, the editors and the reviewers. Any product that may be evaluated in this article, or claim that may be made by its manufacturer, is not guaranteed or endorsed by the publisher.

Copyright (c) 2021 Lou, Ma, LV, Yuan, Xu, Dong, Lin, Tan, Zhang and Chen. This is an open-access article distributed under the terms of the Creative Commons Attribution License (CC BY). The use, distribution or reproduction in other forums is permitted, provided the original author(s) and the copyright owner(s) are credited and that the original publication in this journal is cited, in accordance with accepted academic practice. No use, distribution or reproduction is permitted which does not comply with these terms. 